Review began 01/25/2022 Review ended 01/29/2022 Published 01/31/2022

() Copyright 2022

Ahmed et al. This is an open access article distributed under the terms of the Creative Commons Attribution License CC-BY 4.0., which permits unrestricted use, distribution, and reproduction in any medium, provided the original author and source are credited.

\section{A Cross-Sectional Evaluation of Vitamin D Status and Ovarian Reserve Markers in Subfertile Women: A Single-Center Experience From Pakistan}

Sibtain Ahmed ${ }^{1}$, Ayra Siddiqui ${ }^{2}$, Alinah Qureshi ${ }^{3}$, Syed Sajjad Hussain ${ }^{4}$, Imran Siddiqui ${ }^{1}$, Uzma Imran 1. Pathology and Laboratory Medicine, Aga Khan University, Karachi, PAK 2. Medical College, Aga Khan University, Karachi, PAK 3. Student Life Science Gateway, Mc Master University, Ontario, CAN 4. Head Research and Development, Australian Concept Medical Center, Karachi, PAK 5. Gynecology and Obstetrics, Australian Concept Medical Centre, Karachi, PAK

Corresponding author: Uzma Imran, uzma.imran@aku.edu

\section{Abstract}

\section{Objectives}

This study was conceived with the objective of assessing the correlation between ovarian reserve markers and vitamin D deficiency (VDD) in a selected group of Pakistani subfertile women presenting at a specialized subfertility treatment centre. The measurements of antral follicle count (AFC), serum anti-Müllerian hormone $(\mathrm{AMH})$, serum follicle-stimulating hormone (FSH), and serum vitamin $\mathrm{D}(\mathrm{VD})$ levels were the main tools used for the assessment of ovarian reserve.

\section{Materials and methods}

All female patients aged 18 to 45 years presenting with primary and/or secondary subfertility at the Australian Concept Medical Centre in Karachi, Pakistan from August 2016 to July 2021 were included in the study. The data of all eligible patients were recorded in the pre-defined Performa designed for this study. The Kruskal-Wallis test was applied to report the distribution of the data. The correlation between the categorical variables (25-hydroxyvitamin D [25-OHD] levels with AFC and AMH) was assessed using the chi-square test and Spearman correlation. The comparison was based on vitamin D levels grouped into three categories: deficiency (<20 ng/ml), insufficiency (21-29 ng/ml), and sufficiency ( $>30 \mathrm{ng} / \mathrm{ml})$.

\section{Results}

One hundred ninety-nine cases were evaluated for AFC and hormone analysis. The mean age and BMI were $32.87 \pm 5.49$ years and $28.27 \pm 4.97 \mathrm{~kg} / \mathrm{m}^{2}$. VDD was noted in 127 (68.4\%) cases. No significant difference was noted across BMI, age, duration of subfertility, AMH, and FSH across the VD categories. Moreover, a poor correlation was noted between VD, AMH and FSH on the scatter plot, between VD and FSH $(\mathrm{r}=-0.003, \mathrm{p}=$ $0.966)$ and between VD and AMH $(r=-0.068, \mathrm{p}=0.342)$, respectively.

\section{Conclusions}

This study showed a high frequency of VDD in Pakistani subfertile women, from a specialized subfertility center in the largest metropolis in the country. However, a statistically significant association was not found between the markers of ovarian reserve and VD, showing no ethnic differences in the native Pakistani population. Hence, VD supplementation is unlikely to have an impact on correcting the ovarian reserve status in subfertile women in Pakistan. However, this is a potential area of interest, and evaluation of other indices of reproduction/ovarian reserve and the effect of confounders is required to test this hypothesis longitudinally.

Categories: Endocrinology/Diabetes/Metabolism, Obstetrics/Gynecology, Pathology

Keywords: anti-müllerian hormone, pakistan, subfertility, ovarian reserve, vitamin d

\section{Introduction}

25-Hydroxyvitamin D (25-OHD) is a steroid hormone, rendering an upfront role in regulating the calcium and phosphorous levels in the body. Vitamin D (VD) exists in five different forms, i.e., D1, D2 (ergocalciferol), D3 (cholecalciferol), D4 (dihydroergocalciferol), and D5 (sitocalciferol). In humans, D2 and D3 are more prevalent [1]. Vitamin D deficiency (VDD) is highly prevalent in South Asia, especially in children and pregnant women [2]. The causes of this include limited exposure to sunlight, insufficient levels of VD in the diet, and a higher prevalence of naturally occurring dark skin, which requires a relatively long exposure time for VD synthesis [2,3]. 
VDD is common in women of childbearing age and is often linked to adverse maternal and neonatal outcomes [4]. According to Hogan et al., around 14 million pregnant women are subjected to acute maternal complications globally each year [5]. From a pathophysiological perspective, VD maintains the homeostasis of calcium and phosphate by regulating calcium absorption from the intestine and promoting skeletal utilization. Moreover, VDD is often associated with higher rates of small for gestational age (SGA) and low birth weight neonates and certain subsequent complications that can last into adulthood [6].

Subfertility is estimated to be prevalent at a rate of 12-14\%, with VDD considered to be one of the causative factors [7]. Antral follicle count (AFC), anti-Mullerian hormone (AMH), and follicle-stimulating hormone (FSH) serve as established markers of ovarian reserves. However, AFC values can be subject to possible interobserver or intra-observer variation inherent to sonographic measurements. Therefore, the results might be biased and inaccurately estimated.

On the other hand, AMH, a protein hormone secreted by granulosa cells of the ovaries which regulates early follicle development, is considered a better marker for ovarian reserve due to its standardization and convenience of testing [8]. It is expressed from the onset of puberty until menopause; however, it highly varies in each individual for unidentified reasons. To date, AMH has been found to be unaffected by the stage of the ovarian cycle, unlike FSH [9]. The AMH receptor-II gene promoter is linked with VD-

triggered stimulation. In its active form, VD can upregulate AMH production. Hence, VD serves as a regulator for $\mathrm{AMH}$ concentrations in the blood [10].

Several studies [11,12] have advocated the impact of race and ethnicity on the evaluation of VDD and subfertility. Even though VDD is highly prevalent in Pakistan, there exists a substantial gap in the scientific literature regarding its significance in the female subfertile cohort. Longitudinal studies are required in subfertile women to elucidate the association between VDD and subfertility further. To address gaps in local data, this study was conducted to explore the association between VDD and ovarian reserve via measurements of AFC, AMH, FSH, and 25-OHD in Pakistani women.

\section{Materials And Methods}

A retrospective cross-sectional study was conducted at the Australian Concept Infertility Medical Centre after approval from the institutional ethical review committee (ACIMC-UI-07-2021). All female patients aged 18 to 45 years presenting with primary and/or secondary subfertility at the Australian Concept Medical Centre in Karachi, Pakistan from August 2016 to July 2021 were included in the study $(\mathrm{n}=301)$. Inclusion criteria were focused on subjects labelled as subfertile by the consultant gynecologist, based on the criteria of having failed to conceive after 12 months of no contraceptive use. Patients with missing data, i.e., BMI, duration of subfertility, age, AMH, 25-hydroxyvitamin D (25-OHD), AFC, and FSH were further excluded. Our study was registered with the NIH Clinical Trial Registry (Registration No: NCT05137964). This work has been reported in line with the STROCSS criteria [13].

The biochemical analysis was performed at the section of Chemical Pathology, Department of Pathology and Laboratory Medicine, Aga Khan University, Karachi. 25-OHD was analyzed by a chemiluminescence assay on the liaison XL (DiaSorin) analyzer. AMH was measured using an electro-chemiluminescence assay on the Roche Diagnostic e411 analyzer (Roche, Basel, Switzerland), while FSH quantification was done using the ADVIA Centaur FSH assay from Siemens Medical Solutions Diagnostics USA, Malvern, PA. Internal and external quality assurance were ensured according to the institutional protocol. Moreover, the laboratory is accredited by the College of American Pathologists (CAP) and the Joint Commission International (JCI), ensuring external quality assurance.

AFC was determined through a transvaginal 2D ultrasound of the pelvis. To reduce the bias, all ultrasound scans were conducted in the center using the same machine (Model 6v1: Sonoscape) and vaginal probe (3.5 $\mathrm{Hz}$ ). Before the ultrasound procedure, patients were directed to have an empty bladder, and a standard ultrasound technique was used. Two values were obtained, one for each ovary, and an average was taken to obtain the final value.

The data of all eligible patients were recorded in the pre-defined Performa designed for this study. The Kruskal-Wallis test and one-way ANOVA were applied to report the distribution of the data. The correlation between the categorical variables (25-OHD levels with AFC and AMH) was assessed using the Chi-square test and Spearman correlation. The comparison was based on 25-OHD levels grouped into three categories: deficiency $(<20 \mathrm{ng} / \mathrm{ml})$, insufficiency $(21-29 \mathrm{ng} / \mathrm{ml})$, and sufficiency $(>30 \mathrm{ng} / \mathrm{ml})$ [14]. AMH was categorized as low (<1 ng/ml), low normal (1-2 ng/ml), normal (2-4 ng/ml), and high ( $>4 \mathrm{ng} / \mathrm{ml})$. The third variable, AFC, was also classified into four categories: very low (<6), low normal (6-8), normal (8-10), high normal (10-12), and very high $(>12)$. The p-value $<0.05$, was considered statistically significant. The statistical analysis was performed using the statistical software IBM SPSS Statistics for Windows version 23.0 (IBM Corp. Released 2015, Armonk, NY: IBM Corp).

\section{Results}

A total of 199 cases fulfilled the predefined inclusion and exclusion criteria. VDD was noted in 127 (68.4\%), 


\section{Cureus}

insufficiency in 39 (19.6\%), and sufficiency in 33 (16.6\%) cases. The mean age and BMI of the group were $32.87 \pm 5.49$ years and $28.27 \pm 4.97 \mathrm{~kg} / \mathrm{m}^{2}$ respectively. No significant difference was noted across the BMI, age, duration of subfertility, AMH, and FSH across the 25-OHD categories as shown in Table 1.

\begin{tabular}{|c|c|c|c|c|c|}
\hline \multirow{2}{*}{ Variable } & \multirow{2}{*}{ Total } & \multicolumn{3}{|l|}{ 25-OHD } & \multirow{2}{*}{ p-value } \\
\hline & & Deficiency ( $n=127)$ & Insufficiency (n=39) & Sufficiency $(n=33)$ & \\
\hline Age (years) & $32.87 \pm 5.49$ & $32.69 \pm 5.47$ & $32.23 \pm 5.65$ & $34.30 \pm 5.36$ & 0.235 \\
\hline BMI $\left(\mathrm{kg} / \mathrm{m}^{2}\right)$ & $28.27 \pm 4.97$ & $28.54 \pm 4.99$ & $28.54 \pm 5.29$ & $26.87 \pm 4.38$ & 0.213 \\
\hline Duration of subfertility (years) & $7.75 \pm 4.93$ & $8.06 \pm 5.11$ & $6.74 \pm 4.18$ & $7.76 \pm 5.01$ & 0.349 \\
\hline AMH (ng/ml) & $2.58 \pm 2.49$ & $2.54 \pm 2.36$ & $2.74 \pm 2.88$ & $2.53 \pm 2.59$ & $0.904^{\dagger}$ \\
\hline FSH (mlU/ml) & $8.28 \pm 4.59$ & $8.24 \pm 4.15$ & $9.49 \pm 6.37$ & $7.03 \pm 3.32$ & 0.075 \\
\hline
\end{tabular}

\section{TABLE 1: Comparison of demographic indicators and clinical characteristics with 25-OHD}

AMH: anti-Müllerian hormone, 25-OHD: 25-hydroxy vitamin D, FSH: follicle-stimulating hormone, BMl: body mass index

On further stratification of AMH and AFC into fine grained categories, no significant association was revealed with the 25-OHD subgroup analysis as depicted in Table 2.

\begin{tabular}{|c|c|c|c|c|c|}
\hline \multirow{2}{*}{ Variable } & \multirow{2}{*}{ Category } & \multicolumn{3}{|l|}{ 25-OHD } & \multirow{2}{*}{$p$-value } \\
\hline & & Deficiency n(\%) & Insufficiency n(\%) & Sufficiency n(\%) & \\
\hline \multirow{4}{*}{$\mathrm{AMH}(\mathrm{ng} / \mathrm{ml})$} & Low & $38(61.3)$ & $13(21.0)$ & $11(17.7)$ & \multirow{4}{*}{0.780} \\
\hline & Low normal & $21(65.6)$ & $4(12.5)$ & $7(21.9)$ & \\
\hline & Normal & $47(64.4)$ & 17(23.3) & $9(12.3)$ & \\
\hline & High & $21(65.6)$ & $5(15.6)$ & $6(18.8)$ & \\
\hline \multirow{5}{*}{ AFC } & Very low & $21(72.4)$ & $3(10.3)$ & $5(17.2)$ & \multirow{5}{*}{0.897} \\
\hline & Low normal & 23(54.8) & 10(23.8) & $9(21.4)$ & \\
\hline & Normal & $50(65.8)$ & $15(19.7)$ & $11(14.5)$ & \\
\hline & High normal & $16(64.0)$ & $5(20.0)$ & $4(16.0)$ & \\
\hline & Very high & 17(63.0) & $6(22.2)$ & $4(14.8)$ & \\
\hline \multicolumn{2}{|l|}{ Total } & 127(63.8) & 39(19.6) & 33(16.6) & \\
\hline
\end{tabular}

TABLE 2: Evaluation of AMH and AFC across the 25-OHD subgroups

AMH: anti-Müllerian hormone, 25-OHD: 25-hydroxy vitamin D, FSH: follicle-stimulating hormone

Moreover, a poor correlation was noted between 25-OHD, AMH, and FSH on the scatter plot, between 25OHD and FSH $(r=-0.003, p=0.966)$; and between 25-OHD and AMH $(r=-0.068, p=0.342)$, respectively, as shown in Figures 1-2. 


\section{Cureus}

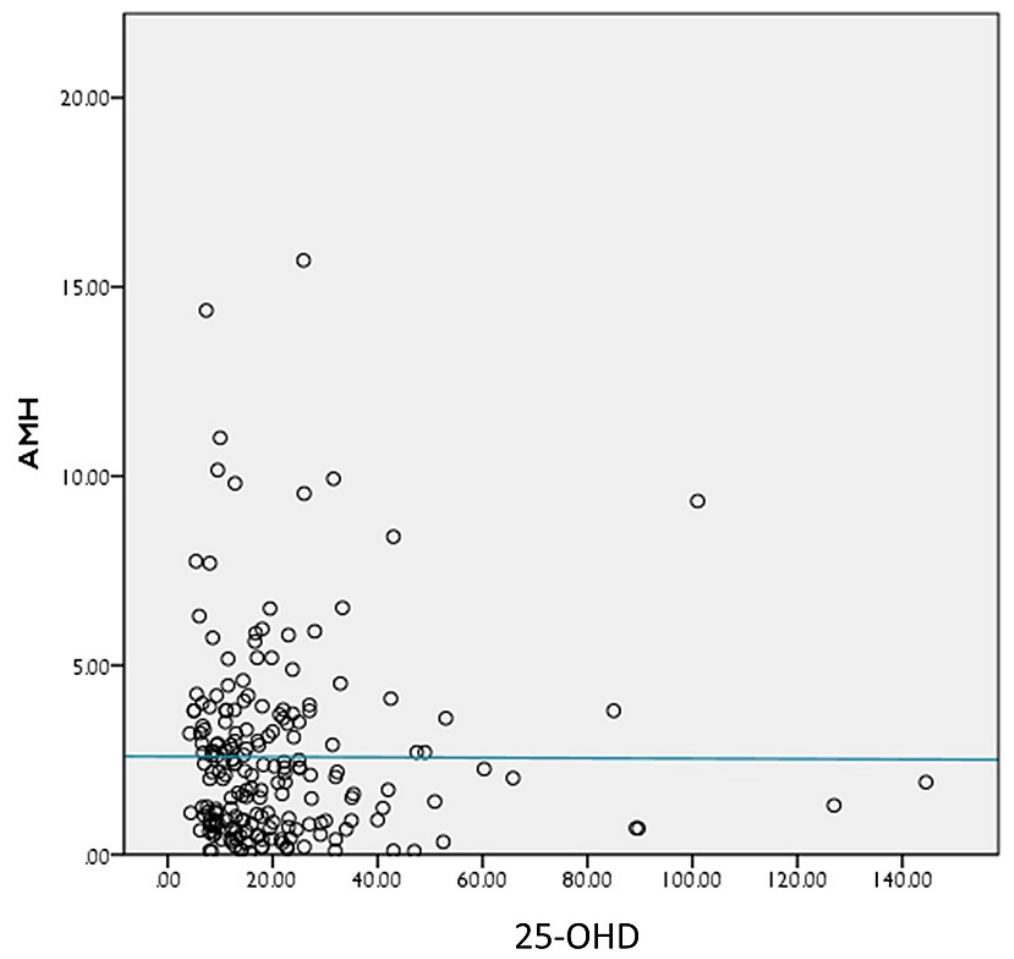

FIGURE 1: Spearman's correlation between 25-OHD and AMH

25-OHD: 25-hydroxy vitamin D, AMH: anti-Müllerian hormone

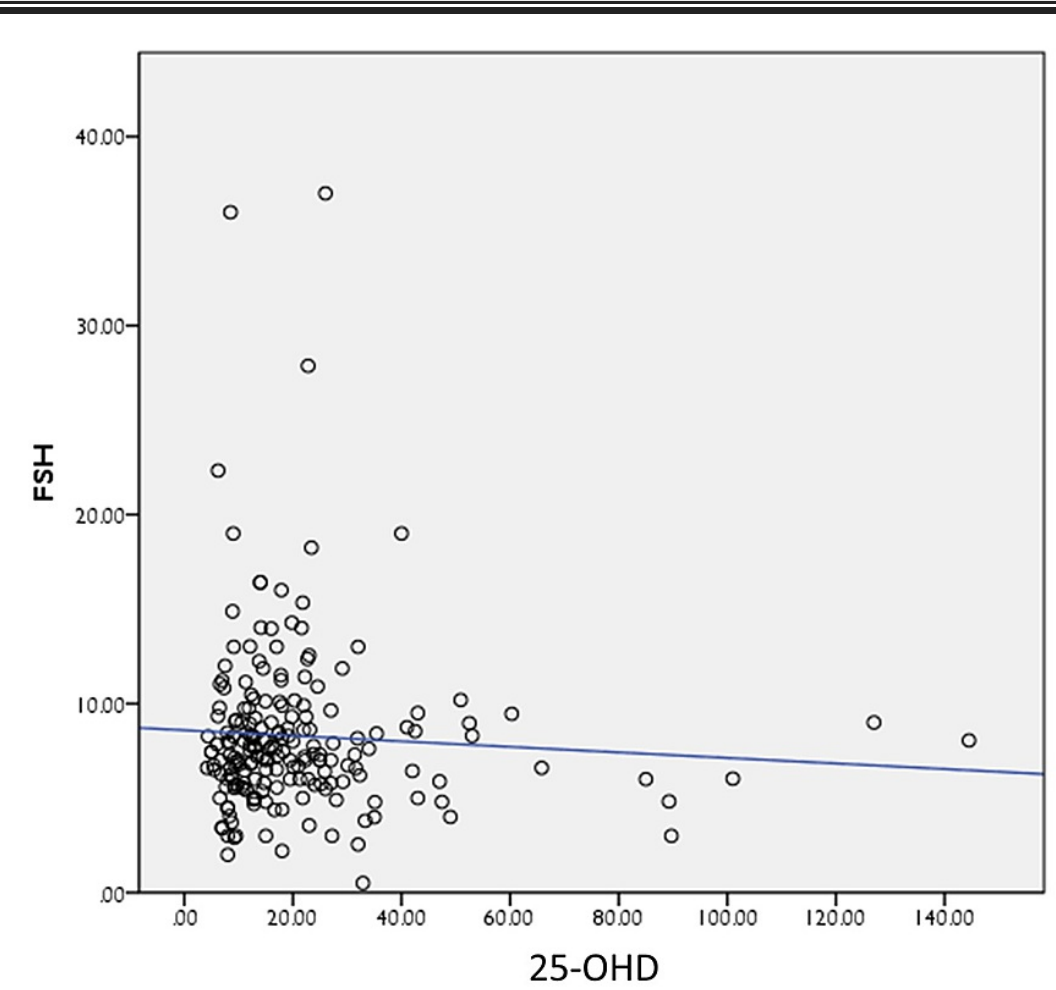

FIGURE 2: Spearman correlation between 25-OHD and FSH

25-OHD: 25-hydroxy vitamin D, FSH: follicle-stimulating hormone 


\section{Discussion}

Our results illustrate that the majority of the study participants were in the deficiency zone (68.4\%). This, in accordance with published literature, demonstrates the high frequency of VDD in Pakistani women in the reproductive age group [15]. A study from Pakistan demonstrated that despite being a year-round sunny environment, VDD has been reported to be alarmingly high among women. A local study group has recorded that more than $90 \%$ of the pre-menopausal women had VDD with values lower than $20 \mathrm{ng} / \mathrm{ml}$ [16]. Similarly, another cross-sectional study from Karachi by Sheikh et al. showed a median concentration of VD as low as $18.8 \mathrm{ng} / \mathrm{ml}$ [17]. VDD in Pakistan has been associated with a lack of sun exposure, especially for women, due to religious obligations, poor dietary intake, and environmental factors, especially pollution [18].

With the growing frequency of subfertility in the metropolis, VDD as a potential contributor can be the missing link in the puzzle. However, a thorough literature review revealed substantial gaps in the literature. Therefore, this study was planned to collect baseline data from a specialized fertility treatment center and assess the relationship between VDD and indicators of ovarian reserve in subfertile women. The AMH levels undertaken in 199 cases correlated well with the AFC measurements.

The regression analysis of the study cohort revealed a poor correlation between subgroups of ovarian reserve biomarkers like AMH and/or AFC and different subgroups of VDD. Moreover, the evaluation of different ethnic groups in Pakistan in relation to VDD was also not able to show any significant correlation. A study by Alavi et al. evaluated 305 subfertile women, referred for in vitro fertilization, showed almost similar results, even after adjusting for baseline factors, authenticating our study findings and consistent with global literature for our part of the world [19]. At this point, regardless of fertility status, finding the VDD so prevalent in our cohort, the authors also felt, on prima facie, that there may be a need to relook and revise the normal reference limit for VD levels among Pakistani women.

The findings can postulate that VD supplementation is unlikely to improve AMH production. As proposed by various molecular characterization studies, VD receptor polymorphism did not lead to subfertility [20-21]. Hence, labelling VDD as a cause of subfertility is unjustifiable. There were certain limitations to this study. First, various environmental and biological factors contributing to subfertility were not considered. Furthermore, only the ovarian reserve markers were evaluated, while the status of other biochemical markers, like thyroid profile, serum prolactin or serum insulin, and complete hormonal status were not considered. Additionally, the impact of seasonal changes on VD levels was not accounted for. Moreover, the retrospective nature of the study and the small sample size may be factors limiting the power of the study. Hence, there is a likelihood that a significant correlation may arise with a larger sample size, emphasizing the need for prospective large-scale multi-center studies.

\section{Conclusions}

This study showed a high frequency of VDD in Pakistani subfertile women, from a specialized subfertility center in the largest metropolis in the country. However, a statistically significant association was not found between the markers of ovarian reserve and VD, showing no ethnic differences in the native Pakistani population. Hence, VD supplementation is unlikely to have an impact on correcting the ovarian reserve status in subfertile women in Pakistan. However, this is a potential area of interest, and evaluation of other indices of reproduction/ovarian reserve and the effect of confounders is required to test this hypothesis longitudinally.

\section{Additional Information Disclosures}

Human subjects: Consent was obtained or waived by all participants in this study. Institutional Ethical Review Committee of the Australian Concept Medical Centre issued approval ACIMC-UI-07-2021. The study was approved by the institutional ethical review committee of the Australian Concept Medical Centre (ACIMC-UI-07-2021). The need for informed consent was waived off as no patient intervention was undertaken and all information was kept anonymized. Animal subjects: All authors have confirmed that this study did not involve animal subjects or tissue. Conflicts of interest: In compliance with the ICMJE uniform disclosure form, all authors declare the following: Payment/services info: All authors have declared that no financial support was received from any organization for the submitted work. Financial relationships: All authors have declared that they have no financial relationships at present or within the previous three years with any organizations that might have an interest in the submitted work. Other relationships: All authors have declared that there are no other relationships or activities that could appear to have influenced the submitted work.

\section{References}

1. Jenkinson C, Taylor AE, Hassan-Smith ZK, Adams JS, Stewart PM, Hewison M, Keevil BG: High throughput LC-MS/MS method for the simultaneous analysis of multiple vitamin D analytes in serum. J Chromatogr B Analyt Technol Biomed Life Sci. 2016, 1014:56-63. 10.1016/j.jchromb.2016.01.049

2. Clemens TL, Adams JS, Henderson SL, Holick MF: Increased skin pigment reduces the capacity of skin to 
synthesise vitamin D3. Lancet. 1982, 1:74-76. 10.1016/s0140-6736(82)90214-8

3. Riaz H, Finlayson AE, Bashir S, Hussain S, Mahmood S, Malik F, Godman B: Prevalence of vitamin D deficiency in Pakistan and implications for the future. Expert Rev Clin Pharmacol. 2016, 9:329-38. 10.1586/17512433.2016.1122519

4. Ni M, Zhang Q, Zhao J, Shen Q, Yao D, Wang T, Liu Z: Relationship between maternal vitamin D status in the first trimester of pregnancy and maternal and neonatal outcomes: a retrospective single center study. BMC Pediatr. 2021, 21:330. 10.1186/s12887-021-02730-z

5. Hogan MC, Foreman KJ, Naghavi M, et al.: Maternal mortality for 181 countries, 1980-2008 a systematic analysis of progress towards millennium development goal 5. Lancet. 2010, 375:1609-1623. 10.1016/S01406736(10)60518-1

6. van der Pligt P, Willcox J, Szymlek-Gay EA, Murray E, Worsley A, Daly RM: Associations of maternal vitamin D deficiency with pregnancy and neonatal complications in developing countries: a systematic review. Nutrients. 2018, 10:10.3390/nu10050640

7. Rudick B, Ingles S, Chung K, Stanczyk F, Paulson R, Bendikson K: Characterizing the influence of vitamin D levels on IVF outcomes. Hum Reprod. 2012, 27:3321-7. 10.1093/humrep/des280

8. Fleming R, Seifer DB, Frattarelli JL, Ruman J: Assessing ovarian response: antral follicle count versus antiMüllerian hormone. Reprod Biomed Online. 2015, 31:486-96. 10.1016/j.rbmo.2015.06.015

9. Dennis NA, Houghton LA, Jones GT, van Rij AM, Morgan K, McLennan IS: The level of serum anti-Müllerian hormone correlates with vitamin D status in men and women but not in boys. J Clin Endocrinol Metab. 2012, 97:2450-5. 10.1210/jc.2012-1213

10. Merhi Z, Doswell A, Krebs K, Cipolla M: Vitamin D alters genes involved in follicular development and steroidogenesis in human cumulus granulosa cells. J Clin Endocrinol Metab. 2014, 99:E1137-45. 10.1210/jc.2013-4161

11. Mitra A, Kundu S, Bhattacharya J, Bhattacharya M: Impact of vitamin D deficiency on IVF outcome in Asian populations. Fertil Steril. 2018, 110:253-254. 10.1016/j.fertnstert.2018.07.724

12. Ozkan S, Jindal S, Greenseid K, Shu J, Zeitlian G, Hickmon C, Pal L: Replete vitamin D stores predict reproductive success following in vitro fertilization. Fertil Steril. 2010, 94:1314-9.

10.1016/j.fertnstert.2009.05.019

13. Agha R, Abdall-Razak A, Crossley E, Dowlut N, Iosifidis C, Mathew G: STROCSS 2019 guideline: strengthening the reporting of cohort studies in surgery. Int J Surg. 2019, 72:156-65. 10.1016/j.ijsu.2019.11.002

14. LeBlanc ES, Zakher B, Daeges M, et al.: Screening for Vitamin D Deficiency: Systematic Review for the U.S. Preventive Services Task Force Recommendation. Agency for Healthcare Research and Quality, Rockville; 2015.

15. Shamsi U, Azam I, Shamsi A, Shamsi D, Callen D: Frequency and determinants of vitamin D deficiency among premenopausal and postmenopausal women in Karachi Pakistan. BMC Womens Health. 2021, 21:194. 10.1186/s12905-021-01339-9

16. Khan AH, Iqbal R, Naureen G, Dar FJ, Ahmed FN: Prevalence of vitamin D deficiency and its correlates: results of a community-based study conducted in Karachi, Pakistan. Arch Osteoporos. 2012, 7:275-82. 10.1007/s11657-012-0108-X

17. Sheikh A, Saeed Z, Jafri SA, Yazdani I, Hussain SA: Vitamin D levels in asymptomatic adults - a population survey in Karachi, Pakistan. PLoS One. 2012, 7 :e33452. 10.1371/journal.pone.0033452

18. Hosseinpanah F, Pour SH, Heibatollahi M, Moghbel N, Asefzade S, Azizi F: The effects of air pollution on vitamin D status in healthy women: a cross sectional study. BMC Public Health. 2010, 10:519. 10.1186/14712458-10-519

19. Alavi N, Ebrahimi M, Akbari-Asbagh F: The effect of vitamin D status on ovarian reserve markers in infertile women: a prospective cross-sectional study. Int J Reprod Biomed. 2020, 18:85-92. 10.18502/ijrm.v18i2.6501

20. Vilarino FL, Bianco B, Lerner TG, Teles JS, Mafra FA, Christofolini DM, Barbosa CP: Analysis of vitamin D receptor gene polymorphisms in women with and without endometriosis. Hum Immunol. 2011, 72:359-63. 10.1016/j.humimm.2011.01.006

21. Hornstein MD: Vitamin D and infertility: the evidence . Fertil Reprod. 2019, 1:31-33. 10.1142/S266131821950004X 